\title{
MOVEMENT ASSISTED COMPONENT BASED SCALABLE FRAMEWORK FOR DISTRIBUTED WIRELESS NETWORKS
}

\author{
Sanjeev Prakash ${ }^{1}$, R. B. Patel ${ }^{2}$ and V. K. Jain ${ }^{3}$ \\ ${ }^{1}$ Department of Computer Science and Engineering, Sant Longowal Institute of \\ Engineering and Technology, Longowal, Punjab, India \\ ${ }^{2}$ Department of Computer Science and Engineering, Chandigarh College of Engineering \\ and Technology, Chandigarh, India \\ ${ }^{3}$ Department of Electrical and Instrumentation Engineering, Sant Longowal Institute of \\ Engineering and Technology, Longowal, Punjab, India
}

\begin{abstract}
Intelligent networks are becoming more enveloping and dwelling a new generation of applications are deployed over the peer-to-peer networks. Intelligent networks are very attractive because of their role in improving the scalability and enhancing performance by enabling direct and real-time communication among the participating network stations. A suitable solution for resource management in distributed wireless systems is required which should support fault-tolerant operations, requested resources (at shortest path), minimize overhead generation during network management, balancing the load distribution between the participating stations and high probability of lookup success and many more. This article presents a Movement Assisted Component Based Scalable Framework (MAC-SF) for the distributed network which manages the distributed wireless resources and applications; monitors the behavior of the distributed wireless applications transparently and attains accurate resource projections, manages the connections between the participating network stations and distributes the active objects in response to the user requests and changing processing and network conditions. This system is also compared with some exiting systems. Results shows that MAC-SF is a better system and can be used in any wireless network.
\end{abstract}

\section{KEYWORDS}

Mobile Agent (MA), Mobile Node (MN), Region Head (RH), Mobile Network Station (MNS), Fixed Network Station (FNS), Network Station (NS)

\section{INTRODUCTION}

Communication networks are capable to integrate a large number of heterogeneous wireless technologies. The invention of mobile devices and wireless applications are affected the performance, scalability, and quality of service (QoS) [25]. The adoption of wireless technologies is due to user requirement, small size of devices with multiple networking interfaces and ability of heterogeneous wireless technologies. The burning issues which are playing major role aremobility, re-addressing, routing, and security. Seamless mobility is one of the main challenges in the existing and future heterogeneous wireless networks [25-28, 30]. Seamless mobility in heterogeneous wireless network needs integration of different network technologies. This integration may facilitate interoperability and convergence among these heterogeneous technologies through the vertical handover (VHO) mechanisms[24]. Heterogeneous wireless networks may comprise of different radio access technologies including GSM, GPRS, HSPA,WLAN, WWLAN, UMTS, WiFi, WiMax and even LTE and to provide high performances by achieving high data rate and supporting multimedia applications, streaming and multicasting with high QoS levels [28]. 
Agent technology offers a new paradigm for distributed computing in which an autonomous program drifts under its own or network station (NS) control from one NS to another NS in a heterogeneous network are called Mobile Agents (MAs) [14]. In other words, a program running at a NS may suspend its execution at an arbitrary point, transfer itself to another NS and resumes execution from the point of suspension. The main areas in which agent technology offers considerable advantages are- distributed heterogeneous network management and information retrieval $[3,20]$. This technology is also offering potential advantages in the area of disconnected computing, also known as wireless or mobile computing and communication, dynamic deployment of code, thin clients or resource-limited devices, personal assistants and agent-based parallel processing $[18,22]$.

This article presents a Movement Assisted Component Based Scalable Framework (MAC-SF) for the distributed network which manages the distributed wireless resources and applications; monitors the behavior of the distributed wireless applications transparently and attains accurate resource projections, manages the connections between the participating network stations and distributes the active objects in response to the user requests and changing processing and network conditions.

Rest of the article is organized as follows. Section 2 gives few most crucial available challenges in wireless networks. Section 3 highlights on System model. Section 4 gives the architecture of MAC-SF. Section 5 highlights on Principle of MAC-SF for Distributed Network Management. Section 6 explores result outcome of network selection process of said system. Section 7 identifies structural difference between Freenet, Gnutella and MAC-SF and Section 8 gives advantages behind the development of MAC-SF. Finally article is concluded in Section 9.

\section{Challenges}

The Internet started out as a fully symmetric, heterogeneous network of cooperating users. It is growing for accommodating its millions of users flocking online, technologies have been put in place that have split the network into a distributed system with relatively few servers and large number of users [23]. These facts pretence challenges and barriers for the distributed wireless network applications. This shows that design of both the network and the applications are bicycle. Application developers must design healthy applications that can function in the complex wide area network environment, and network designers must build in capabilities to handle new distributed wireless applications $[1,2,4,5]$. The experience of many issues in the early Internet may be brought forward to design tomorrow's systems.

Distributed heterogeneous wireless networks are usually large-scale dynamic systems whose NSs are distributed over wide geographic area [6]. The NSs can join and leave continually in distributed wireless systems which generates dynamic system with high rate of roil and changeable topology [29]. In this system resources or nodes are restricted to temporary availability only and normally nodes may disappear at a given time from the network and reappear at another location in the network with an unpredictable pattern. Thus in dynamic system, one of the most challenging problem is to manage the network so that resources may always successfully be located by their requesters when needed [6-8].

Mobility is an event taking place whenever a mobile node (MN) moves from one wireless cell to another and change the point of attachment without affecting the access services. There are two kind of events take place in managing seamless connectivity of the MNs in the network viz., horizontal and vertical handoffs. A handover occurs within the same wireless access technology the process is known as horizontal handoff and when takes place between different wireless 
access technologies known as vertical handoff. Initiation of vertical handoff in the heterogeneous networks takes place for convenience rather than connectivity reasons [24]. It is one of the crucial issues in mobile wireless communication technologies. Since seamless connectivity is required for a user when moves from one location to another. The researchers are using different parameters to provide the solution of vertical handover.[32-37].

In order to facilitate resource awareness in such a large-scale dynamic distributed heterogeneous wireless environment, a specific resource management strategy is required which is taking into account distributed wireless system's characteristics as a main parameter. Still there are many other challenges those must be considered before the grand success [12-13, 31]. To address the above issues a management system is needed for distributed heterogeneous wireless networks which uses probabilistic algorithms for increasing the anonymity and thus origins cannot be easily tracked by analyzing network traffic. Therefore distributed wireless networks will re-issue computation jobs to other participants to ensure that work is not lost if earlier participants drop out of the network while they were performing a computation step. A distributed wireless network will typically not rely on established infrastructure [15-17, 19].

\section{SYSTEM MODEL}

A middleware is measured as a necessary layer among the hardware, operating environments and applications. The middleware layer provides an appropriate interface to various applications, a runtime environment that supports and coordinates multiple applications. A middleware is often used by traditional systems as a bridge between the operating environments and the applications. It makes the development of distributed wireless applications possible. A traditional distributed wireless middleware is not adequate for the heterogeneous wireless network's requirements of memory, energy, and computing power. Mechanisms are required to achieve adaptive and efficient use of system resources. The maintenance of traditional middleware architectures is also not easy due to the ad hoc constraints. For these kinds of networks, a middleware that is simple, light, and easy to implement is needed. Thus, we are required to develop a computing/communication distributed wireless systems that fulfils most of the above the challenges.

\section{SYSTEM ARCHITECTURE}

This article take up above identified challenges and propose a middleware known as "Movement Assisted Component Based Scalable Framework (MAC-SF)" for distributed heterogeneous wireless networks as shown Figure 1. MAC-SF supports mobile entity heterogeneity. A application developer writes an MA code as per need of an application. A MN will be the member of a region. A $\mathrm{MN}$ in a region will work like region head (RH) and maintains information about other members in that region. It keeps an intelligent for gathering and maintaining the information about the network. RH also keeps a MA for finding the network topology[14]. When a mobile node desires to search some information it requests to RH for members information (viz., IP address, identification certificate, etc.). If a RH is not aware about availability of the type of services a asking $\mathrm{MN}$ is interested and presence of the same in the region. It facilitates the same to the $\mathrm{MN}$ and $\mathrm{MN}$ creates a MA as per the need of the application to perform its desired task in the present region. Further, if the requesting task is not completed with the help of members in the present region then MN moves to next region or it may take help of the RH and multicast its MA to the RHs in the network. After completion of the task final result reaches to the MN via multipath which was its launching station. In MAC-SF system it is assumed that wireless network consist any number of Mobile Network Stations (MNSs)/Fixed 
Network Station (FNS). A MNS/FNS may often be disconnected for prolonged period of time due to the low power of battery or out of coverage area of network signal but may also be frequently reallocated between different wireless networks at different time.

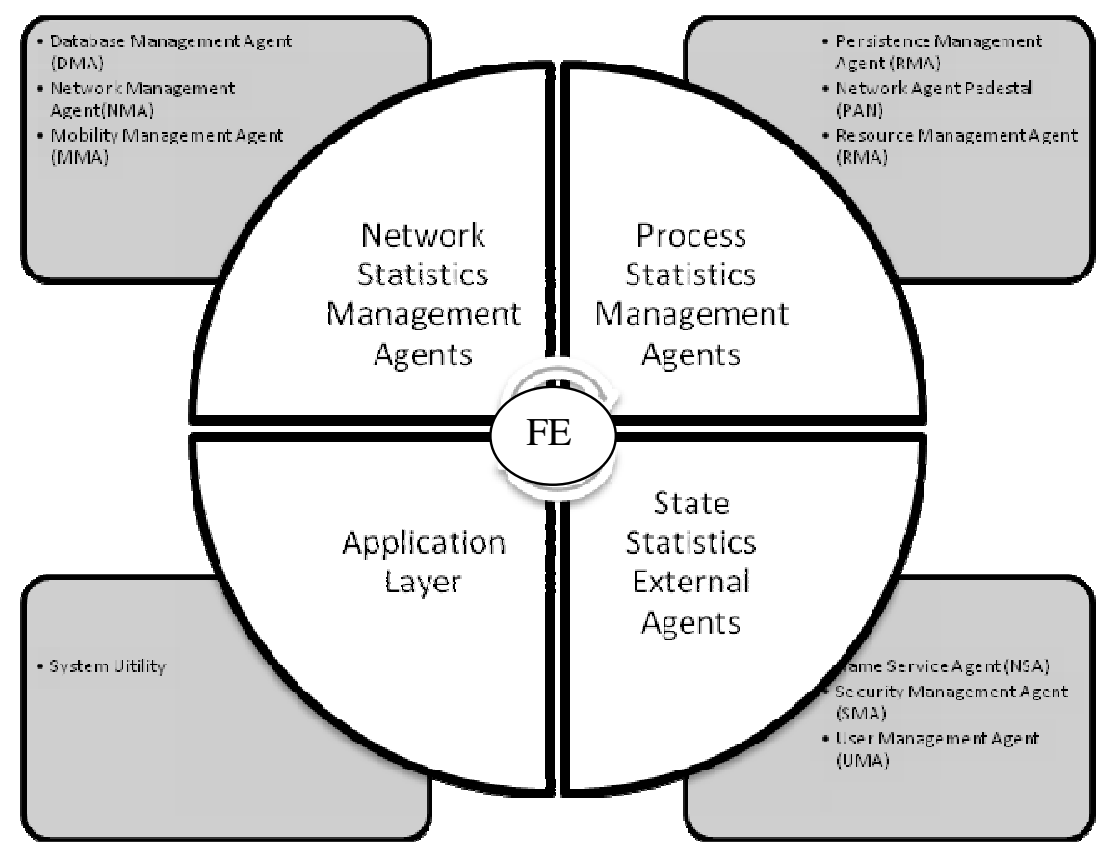

Figure 1. System Architecture of MAC-SF

In wireless communication and computing environment users do not require to maintain a fixed and universally known position in the network and enables unrestricted mobility of the MNSs. There may be any numbers of distributed wireless networks in the distributed system, which communicate through the existing wireless ad hoc network infrastructure. It is suggested that there should be a MNS in between different networks for providing mobility to the heterogeneous MN among heterogeneous networks.

MAC-SF serves users at anytime, anywhere. It consists of the agents modules and the forwarding Engine. The synergy is provided by a well established, unified baseline architecture that promotes the development of efficient, secure and fault tolerance wireless computing applications.

\subsection{Forwarding Engine (FE)}

The FE is the basic utility module, which lies below the agents modules and is responsible for driving the MAC-SF, by ensuring proper coordination between the various agents and making them work in tandem. It uses secure socket layer (SSL) protocol secured communication pipe to transfer requests to/from other MAC-SFs.

Agents are categories into three groups- Network Statistics, Process Statistics and External Process/Event Statistics Agents as per their requirement. Various agent modules help to perform functions like- forwarding/receiving packets, communication, mobility, name services, adaptation, fault tolerance, handoff, etc. 


\subsection{Process Statistics Management Agents}

These agents are used to maintain the states of different processes, viz., Mobile devices, MAs, and other processes related to MAC-SF. This work is distributed with the help of three agentsPersistence Management Agent (PMA), Network Agent Pedestal (PAN), and Resource Management Agent (RMA). PAN works like a multiagent system to design, control and manage the MAs.

Resource Management Agent (RMA): The overall network load among different nodes, a RMA manages the resources of the system (viz., system memory used by MNS/FNS/MA, files, etc.), and also controls the admission of MNs and MAs that subscribe the services. Main responsibilities include: authorization \& authentication, Type of Services (ToS) and QoS negotiation, subscription and un-subscription of service. To simplify the functionalities RMA is subdivided into two another modules Resource Allocator Agent (RAA) and Replication Controller Agent (RCA). RAA manages the resources for newly subscribed services. Resources are assigned fairly among MNs/MAs and at the same time fulfilling individual MNs/MAs requirement. $\mathrm{RC}$ keeps track of the global state of the distributed resources consistent among all local resources based on a given coherence strategy. A user is free to use MAs or MN both or only MN as per need of the applications.

Persistence Management Agent (PMA): PMA is implemented for recovering the lost information in between disconnection during performing the computations using MAs. It also provides fault tolerance to the communication channels on failure. The execution environment for both public architecture services and application specific architecture services are provided by the core module of the architecture, which is known as FE with the help of different agents running just above.

Network Agent Pedestal (PAN): It is developed for managing the heterogeneous networks system fault tolerance and load balancing on the network. It mainly contains five MAs and two servers which are governed under the flagship of PAN. Further as per need of applications any number of stationary and MAs can be added to the system. PAN works as multiagent system. It permits application developer to design and implement his/her own agent as per need of the application, i.e., PAN is scalable and adaptive components in MAC-SF.

\subsection{External Process/Event Statistics Management Agents}

These agents deal with exterior issues, i.e., external communications-send/receive request(s). This work is carried with the help of three agents- Security Management Agent (SMA), Name Service Agent (NSA), and User Management Agent (UMA).

User Management Agent (UMA): When a MNS/FNS communicates with some other MNS/FNS for the fulfilling the requirements for some required resources which may be available on that MNS/FNS. To make this type of communication user friendly User Interface (UI) is provided. It run on a MNS/FNS and enables the mobile applications to communicate with other MNSs/FNSs in the network. UI is platform independent and adapts variety of MNSs/FNSs displays.

Name Service Agent (NSA): Names are detached from their corresponding objects: an MN/MA may possess a name without having immediate access to other MN/MA of that name. To enable mobility and disconnected operation, all MNS/FNS/MA/MN across a wireless network should be denoted by unique names. NSA manages the process of naming and identification. 
During this process there is very much risk of security and inconsistency in the data due disconnection and security.

Security Management Agent (SMA): It assumes $n$ number of mobile MNSs/FNSs. SMA coordinates among the applications and collaborates with NSA because names are symbolic ways of referring to them across a network. It addresses Security, trust and privacy from the very beginning of system design viz., hardware, operating system, protocols, and architecture. It protect RH against unauthorized modifications, program validation/verification trust modelling, sharing fragmented information in efficient and controlled manner, Key/certificate management, and implications of ad-hoc communities.

\subsection{Network Statistics Management Agents (NSMAs)}

To establish the communication link among the MNS/FNSs and BHs (running FE of the MAC$\mathrm{SF}$ at the RH of a region UI calls the NSMA which manages the data exchange between the different components of the MAC-SF. To access the necessary information from the networks MNSs/FNSs and MAs use different types of communication channels. The NSMA decides which network component will perform desired task efficiently at lower cost. It also supports the adaptivity of the system in case of bandwidth fluctuation. This is assisted by three agents.

Mobility Management Agent (MMA): It performs the Location Management (LM) of the MNS/FNSs as well as of MAs. LM enables the system to track the locations of MNS/FNSs/MAs between consecutive communications. It includes two major tasks- location registration/location update and call delivery. The MNS/FNS/MA periodically informs RH to update relevant location databases with its up to date location information. The system determines the current location of the MNS/FNS/MA based on the information available at the RH databases before initiating a communication for the MNS/FNS/MA. The design of LM techniques has the following objectives: (1) Reduction of latency of service delivery. (2) Quality of service (QoS) guarantees in different RHs. (3) When the service areas of heterogeneous wireless networks are fully overlapped: (a) through which RHs a MNS/FNS/MA should perform location registrations. (b) In which RHs and how the up-to-date user location information should be stored. (c) How the exact location of a MNS/FNS/MA would be determined within a specific time constraint. The LM keeps MNS/FNS connection active when it moves from one RH to another. When a MN is moving out of the serving area of a RH will enter another overlaying RH shortly. If a MNS/FNS is connected to a particular RH and wants chooses to be handed-off to the underlying RH for its future service needs may do it.

Network management Agent (NMA): It identifies the topology of the network. NMA uses servers of PAN for facilitating distributed environment for adapting the nature of the network bandwidth.

Database Management Agent (DMA): It maintains information for the clients, packets, user information, security database, etc.

\subsection{Application Layer}

This layer is specially designed to create network applications. This layer considers all other components of the system as a resource. For making the communication over the network this makes the communication through the FE. 


\section{MAC-SF Principle For Distributed Network MANAGEMENT}

Heterogeneous wireless networks generally comprise of a fixed backbone network and a wireless network. Mainly three distinct sets of entities play the key role in the system, viz., MNs, MNSs and FNSs. A NS that moves while retaining its network connection is called a MNS. The fixed network comprises of the FNSs and the communication links between them. Some of the FNSs are called base station (BS). These are augmented with a wireless interface, and provide a gateway for communication between the wireless network and the fixed network. Due to the limited range of wireless transreceivers, a MNS/MN can communicate with a BS. The geographical area covered under a region is a function of the medium used for wireless communication. Currently, the average size of a region is of the order of 1-2 miles in diameter. In the constantly changing environment demand for services may increase, then for maintaining the required grade of services regions are splitted to handle the traffic increased without increasing the bandwidth of the system. A MN communicates with one BS at any given time. The BS is responsible for forwarding data between the MNS/MN and the fixed network. Due to mobility, MNS/MN may cross the boundary between two regions while being active. The task of forwarding data between the fixed network and the MNS/MN are routed through the new regions. This process is known as handoff[40-41]. The initiative for a handoff can come from the MNS or the BSs. It helps to maintain an end-to-end connectivity in the dynamically reconfigurable network topology.

MAC-SF works on some assumptions for the distributed heterogeneous environment. Figure 2 presents setup of the MAC-SF based system. It assumes that the global wireless mobile communication network is divided into network domains (mobile switching centre-MSC), regions (subnetworks, i.e., base switching centre (BSC) controlled network) and MNSs. MSC works as domain management server (DMS) in each network domain which has information about all other DMSs in the global wireless mobile communication network. It also maintains information about all the BSCs, i.e., regions in the network domain. It is responsible for maintaining uniqueness of names of BSCs (regions), which are part of that network and helps to identify the region/BSC in/under which MNS/MN is present.

Each DMS maintains a Domain Mobile Node Database (DMND), for information about the current location of all MNs which were registered in that domain or transited though. Every region (BSC) maintains information about all base stations (BSs)/MNSs which are part of that region. A MN can be a member of an existing MSC/BSC covered area/region or can start in a new MSC/BSC region. In each region, a Region Mobile Node Database (RMND) is present at a BS which runs at the gateway (BSC) of a subnetwork. It contains location information about each MNS/MN which was registered in that region or transited through it. This NS acts as the Mobile Node Name Server (MN2S), which manages the RMND. RMN2S is responsible for maintaining uniqueness of names of all MNSs/MNs, registered in that region. When a new MNS/MN is registered, the user assigns a name to it by registering in the RMND of its birth region.

DMND stores the information in the form $(M N, F D, r)$. It represents that a $M N$ may be found in the region $r$ of the foreign network domain $F D$, or transited through. Each entry of RMND of the form $(M N, r, N i l)$ represents the region $r$ where $M N$ is found or transited through. Similarly $(M N, N i l, M N S)$ represents a $M N$ which exists in that region at a BS/MNS. For DMND and RMND, the primary key is the name of $M N$. 
MN movement from one network domain to another is always accomplished through the DMS. During inter domain migration the MN has to update location information in the DMND of the present domain and register in the DMND of the target network domain.

For intra region migration, it updates its location information in the RMND of the region. This is an Intra Region Location Update. When inter region migration takes place, the MN updates the location information in the RMND of present region and register in the RMND of the target region, specifying the BS/MNS in that region to which it is migrating. The location protocol used for managing the MNs deals with three processes: name binding, migration and location, each related to a particular phase in the MN's lifetime. We have defined four atomic operations on DMND and RMND.

- bind process is performed when a name is assigned to a newly registered MN, whose birth location is also stored. This process causes the insertion of a new tuple in the database. As the MN name has to be unique, this operation fails if a tuple with the same name already exists in the database.

- newloc process is performed when the MN changes its location, by migrating to a new one. This process updates the tuple already present in the database.

- find process is performed when a $\mathrm{MN}$ has to be located in order to interact with it. For a given $\mathrm{MN}$ name, this process returns the current location of the $\mathrm{MN}$.

- unbind process is performed when a MN name is no longer used (i.e., the MN has been disposed off). This process causes the deletion of the relative tuple from the database.

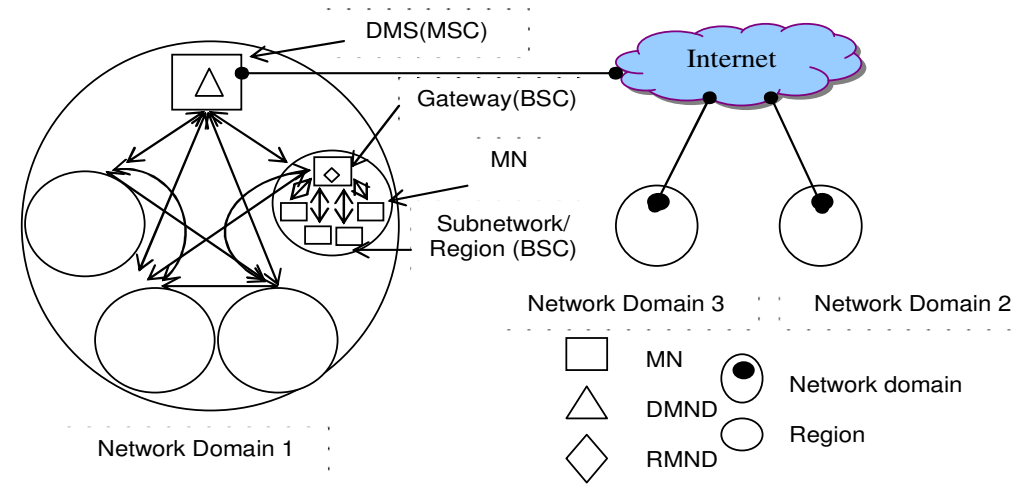

Figure 2. Wireless Network Environment Distribution Policy for MAC-SF

Each DMS, BSC, BS and MNS is equipped an intelligent agent and a MA for maintaining network topology and current status of its neighbouring stations. Normally agents observed technique for order preference by similarity to the ideal solution (TOPSIS) [42] for getting the services, network path from one point to another. In TOPSIS the best alternative have shortest distance from the positive ideal solution and the farthest distance from the negative ideal solution [3]. A matrix is constructed on the base of set of alternative and corresponding attributes (criteria). In this method it is assumed that there are $A_{m}$ alternatives and $C_{m}$ criteria. A series of steps of TOPSIS are as described below:

Step 1: Construct a matrix of value assigned to alternatives corresponding to criteria.

Step 2: Weight vector $\left(\mathrm{w}_{\mathrm{j}}\right)$ for each attribute is computed and is as follows. 
International Journal on Computational Science \& Applications (IJCSA) Vol.5, No.5,October 2015

$$
\sum_{j}^{n} w_{j}=1, \quad j=1,2, \ldots, n
$$

Step 3: Construct the normalized decision matrix. Each element $r_{i j}$ of the Euclidean normalized decision matrix may be computed as

$$
\mathrm{A}=\left[r_{i, j}\right]_{\mathrm{m} \times \mathrm{n}}, \mathrm{r}_{\mathrm{i}, \mathrm{j}}=\frac{x_{i, j}}{\sqrt{\sum_{i=1}^{m} x_{l, !}}} ; \quad \text { for } i-1,2, \ldots, m ; \mathrm{j}=1,2, \ldots, \mathrm{n} .
$$

Step 4: The weighted normalized decision matrix is obtained using the following.

$$
V_{i, j}-w_{i, j} * r_{i, j} ; \quad i-1,2, \ldots, m ; \mathbf{j}-1,2, \ldots, \mathbf{n} .
$$

Step 5: Positive and negative ideal solutions are determined by the following expression.

$$
\begin{aligned}
A^{+} & =\left\{\left(\max _{i}\left\{v_{i, j}\right\} \mid j \in B\right),\left(\min _{i}\left\{v_{i, j}\right\} \mid j \in C\right)\right\} \\
& =\left(v_{1}^{+}, v_{2}^{+}, \ldots v_{m}^{+}\right)
\end{aligned}
$$

where $\mathrm{B}$ associated with benefit criteria and $\mathrm{C}$ associated with cost criteria.

Step 6: Calculate the ideal separation measurement. The positive and negative ideal separation of each alternative is calculated by the Euclidean distance:

Positive Ideal Separation

$$
S_{l}^{+}-\sqrt{\sum_{j=1}^{n}\left(v_{t_{j}}-v_{j}^{+}\right)^{2}} ; \quad i-1,2, \ldots m .
$$

Negative Ideal Separation

$$
S_{i}^{-}=\sqrt{\sum_{j=1}^{n}\left(v_{i, j}-v_{j}^{-}\right)^{2}} ; i=1,2, \ldots m .
$$

Step 7: A relative distance of each alternative from ideal and negative ideal solution is computed using the following formula.

$$
C_{i}=\frac{S_{i}^{-}}{S_{i}^{+} \mid S_{i}^{-}} ; i=1,2, \ldots i n .
$$

Step 8: Arrange set of alternatives in descending order. Highest value is the best and lowest value is the worst. 


\section{Simulation And Performance Study}

In the simulation, there are four candidate networks and each networks with six attributes associated in heterogeneous wireless networks environment. Three different technologies are used such as two UMTS networks, one WiMAX and one WLAN. We have used- Packet Delay, Packet Loss, Available Bandwidth, Network Load, Security ) and Cost attributes for the simulation. These attribute values are shown in Table 1.

Table 1. Attributes of network

\begin{tabular}{|l|l|l|l|l|l|l|}
\hline $\begin{array}{l}\text { Criteria } \\
\text { Technology }\end{array}$ & $\begin{array}{l}\text { Delay(D) } \\
(\mathrm{ms})\end{array}$ & Packet Loss & $\begin{array}{l}\text { Available } \\
\text { Bandwidth } \\
(\mathrm{Mbps})\end{array}$ & $\begin{array}{l}\text { Network } \\
\text { Load }\end{array}$ & Security & Cost \\
\hline UMTS-1 & 35 & 70 & 0.0122 & 15 & 80 & 0.6 \\
\hline UMTS-2 & 30 & 40 & 0.0122 & 0 & 80 & 0.8 \\
\hline WiMAX & 60 & 30 & 2.0 & 20 & 70 & 0.7 \\
\hline WLAN & 15 & 15 & 0.30 & 0 & 60 & 0.1 \\
\hline
\end{tabular}

Using TOPSIS method we are getting the following normalized decision matrix $r_{i, j}$ where value of $i$ is available alternative network varying from 1 to 4 and $j$ is varying from 1 to 6 and is corresponding network attribute. The matrix is given below.

\begin{tabular}{|l|l|l|l|l|l|}
\hline 0.453743 & 0.641016 & 0.006032 & 0.6 & 0.548151 & 0.489898 \\
\hline 0.388922 & 0.73259 & 0.006032 & 0 & 0.548151 & 0.653197 \\
\hline 0.777844 & 0.183147 & 0.9889 & 0.8 & 0.479632 & 0.571548 \\
\hline 0.194461 & 0.137361 & 0.148335 & 0 & 0.411113 & 0.08165 \\
\hline
\end{tabular}

The weighted normalized decision matrix $\left(\mathrm{V}_{\mathrm{i}, \mathrm{i}}\right)$ where value of $i$ is available alternative network varying from 1 to 4 and $j$ is varying from 1 to 6 and is corresponding network attribute. The

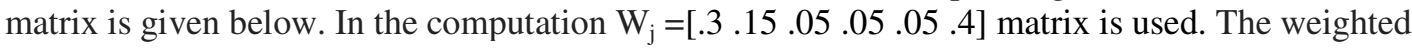
normalized decision matrix is given below.

\begin{tabular}{|l|l|l|l|l|l|}
\hline 0.136123 & 0.096152 & 0.000302 & 0.03 & 0.027408 & 0.195959 \\
\hline 0.116677 & 0.109888 & 0.000302 & 0 & 0.027408 & 0.261279 \\
\hline 0.233353 & 0.027472 & 0.049445 & 0.04 & 0.023982 & 0.228619 \\
\hline 0.058338 & 0.020604 & 0.007417 & 0 & 0.020556 & 0.03266 \\
\hline
\end{tabular}

Positive and Negative Ideal Solutions are
$\mathrm{A}^{+}=[0.197038$
0.264376
0.212612
$0.204976]$
$\mathrm{A}^{-}=[0.130275$
0.106873
0.189131
0.228729] 
A relative distance of each alternative network from positive ideal and negative ideal solution is as follows.

$\mathrm{C}=\left[\begin{array}{l}0.3980120 .2878740 .4707760 .527384\end{array}\right]$

UMTS- $1=0.398012$, UMTS-2 $=0.287874$, WiMAX $=0.470776$, WLAN $=0.527384$

Since, WLAN yields highest value, thus it is best network for selection. It is reasonably good value for identifying WLAN as the best network. This network is more useful and fulfils the demands of the users in better way. Normally WLAN uses the following metrics- delay, packet loss, overhead and bandwidth allocation, etc.

\section{A CoMParison Of Freenet [10], GNuTella [38-39], AND MAC-}

In this section we compare MAC-SF with popular distributed system, viz., Freenet and Gnutella. These systems are specially designed for resource management on wide area networks. But it is not possible to use them on wireless heterogeneous networks. In the following wide area network properties of MAC-SF are compared with Freenet and Gnutella.

The Freenet network consists of many active and passive devices (known as NS) on the Internet running Freenet protocol and together these NSs form a large network. Each NS communicates with one another and also play the role of routing NSs ( i.e., dual role means as client/server). Searching and distribution of data is handled entirely by NSs. Each NS is basically equal in function and capability. There does not exist a NS that is conscience of the entire network. Freenet uses its own protocol and is implemented on a network with a complex network topology, much like Internet Protocol (IP). Each NS knows only about its some neighbours that it can reach directly. This property is available in each NS. A user first discovers the address of one or more existing NSs through out-of-bound means and then, starts to sending messages and finally joins a Freenet network. Freenet is designed with the following objectives: Ambiguity for both producers and consumers of information. (b) Plausible deniability for stored of information. (c) Resistance to attempts by third parties to deny access to information. (d)Efficient dynamic storage and routing information. (e) Decentralization most of the network functions.

The Gnutella network consists of many NSs on the wide area network running a Gnutella "servant" application that allow them to essentially act as both servers/clients. Each NS has the capability to connect to other NSs to form a private network or can join one of the general public networks. This is done by connecting to a known NS on the network. All requests are sent through this particular NS to all of the NSs it is aware of, i.e., each NS in Gnutella uses HTTP and keeps information about its neighbours. There are many different applications employing Gnutella protocols. It is designed for the following goals: (a) Being a fully distributed information-sharing technology. (b) Avoid the use of portals, which today are stuffed with advertisements. (c) To act as a mini search engine and file serving system in one.

The MAC-SF network consists of many NSs on the Internet running a MAC-SF "FE" that allow them to essentially act as both servers/clients. Each NS has the capability to connect to other NSs and act as router to form a private network or they can join one of the general public networks. There are no dedicated clients/servers. This is done by connecting to a known NS on the network. All requests are sent through this particular NS to all of the NSs it is aware of, i.e., each node maintains topology of the network. This NS also works as an authentication centre if private information is to be shared between two NSs. MAC-SF uses HTTP and Mobile Agent Paradigm (MAP) for searching and distribution of information and it is handled entirely by NSs. There does not exist a NS that is conscience of the entire network because each NS has equal role in the 
network. MAC-SF uses its own port and protocol and is implemented on a network with a complex network topology. Before joining a MAC-SF network, a user needs to first discover the address of one or more existing NSs through out-of-bound means and then start to send messages. MAC-SF protocols generally have the following design goals. (a) Fully distributed information sharing technology. (b) Avoid the use of portals, which today are stuffed with advertisements. (c) MA mini search engine and file serving system in one which acts green energy system.

Freenet, Gnutella and MAC-SF each store data differently. Every Freenet NS holds three types of information: key, reference, and data (optional). The key (binary file key) is a unique identifier for data posted by Freenet NSs obtained by applying a hash function. Each NS stores many keys. The reference information refers to addresses of other Freenet NSs that are likely to know about the keys stored in the particular NS. The data information is simply the data corresponding to the keys. Gnutella and MAC-SF do not utilize a data storing system similar Freenet does. Any posted file is identified by its actual file name given by the source NS.

Freenet, Gnutella and MAC-SF retrieve and return data in different ways as well. There are two scenarios to consider concerning Freenet's manner of data retrieval: satisfying or not satisfying the request. For example, if $\mathrm{NS}_{1}$ is the requesting $\mathrm{NS}, \mathrm{NS}_{2}$ is destination $\mathrm{NS}, \mathrm{NS}_{3}$ is reference $\mathrm{NS}$ of $\mathrm{NS}_{2}$, and $\mathrm{NS}_{4}$ is a secondary NS connected to $\mathrm{NS}_{2}$. If $\mathrm{NS}_{2}$ has the requested data then it sends to $\mathrm{NS}_{1}$, but does not forward the request to any other NSs. Also, the returned data is exactly that of the requested key. In addition, random search engine-style searches are currently not possible in Freenet and a random search engine-style searches are also possible in MAC-SF. It permits this with the help of MAP. Further MAC-SF does not nuisance for type of network is being in use.

The following is the ill-fated scenario, if $\mathrm{NS}_{2}$ does not have the requested data, but has the reference address then $\mathrm{NS}_{2}$ forwards the request to the reference address $\mathrm{NS}_{3} . \mathrm{NS}_{3}$ repeats this process either the key is found or a number of hops is attained to indicate failure. If $\mathrm{NS}_{2}$ has neither the data nor the reference address, then the NS will give the key closest to the requested one as the information is likely to be related to the requested one.

In a Gnutella network, NSs interact with each other using five different message types including Ping, Pong, Query, Query Response, and Get/Push. If $\mathrm{NS}_{1}$ makes a request to $\mathrm{NS}_{2}, \mathrm{NS}_{2}$ sends $\mathrm{NS}_{1}$ any relevant data and pass the request to $\mathrm{NS}_{3}$. Also, $\mathrm{NS}_{2}$ takes note that $\mathrm{NS}_{1}$ has made the particular request. If $\mathrm{NS}_{3}$ also has relevant data pertaining to the request, then it sends a notice that it has the data to $\mathrm{NS}_{2} . \mathrm{NS}_{2}$ recalls that the notice is meant for $\mathrm{NS}_{1}$ and sends the notice to $\mathrm{NS}_{1} . \mathrm{NS}_{1}$ can then establish a indirect connection to $\mathrm{NS}_{3}$ for the relevant data. If $\mathrm{NS}_{2}$ cannot satisfy the request, a multicast request is sent to all NSs on the particular network. In addition, the returned data could be an entire file, but is not limited to just that. The return could be an URL or anything else pertinent. This is contrary to Freenet's return of only the requested key.

In a MAC-SF network, NSs interact with each other using four different message types including Ping, Query, Query Response, and Get/Push. If $\mathrm{NS}_{1}$ makes a request to $\mathrm{NS}_{2}, \mathrm{NS}_{2}$ will send $\mathrm{NS}_{1}$ any relevant data and pass the request to $\mathrm{NS}_{3}$. Also, $\mathrm{NS}_{2}$ takes note that $\mathrm{NS}_{1}$ has made the particular request. If $\mathrm{NS}_{3}$ also has relevant data pertaining to the request, then it sends a notice that it has the data to $\mathrm{NS}_{2}$ and also sends the notice to $\mathrm{NS}_{1} . \mathrm{NS}_{1}$ can then establish a direct connection to $\mathrm{NS}_{3}$ for the relevant data. If $\mathrm{NS}_{2}$ cannot satisfy the request, a multicast request is sent to all NSs in the region. In addition, the returned data could be an entire file, but is not limited to just that. This is contrary to Freenet's return of only the requested key. 
When search data is big in size and it is not possible to carry an agent then the agent clones itself, so the whole data may be transported across the network. The cloned agents transport the data on the network safely. For data service MAC-SF is safe and efficient, because agent carries sensitive data in a sealed box [14].

A major difference between Freenet, Gnutella and MAC-SF is the processes employed when a request is returned and the gains from using these processes. For satisfying the requester a Freenet NS passes the entire data to the requester. This is optional in Gnutella and MAC-SF. In Freenet network when the data passes through multiple NSs back to requester, each NS keeps a copy (within a reasonable size limit). A NS keeps the data as long as other NSs are requesting it, but discard the data after some time when there finish being requests for it. It also brings data closer to users who want it. Once the intermediary NSs have copies of the data, subsequent requests for the data will be satisfied quicker and more directly. When a NS finds that it is receiving a lot of fulfilled requests routinely from one of its closer NSs, it tends to favour that NS for future requests. This has the effect of increasing bandwidth for the users but compromise the security. This replication is limited in case of MAC-SF.

The level of anonymity for users also differs in Freenet, Gnutella and MAC-SF. In Freenet, users can distribute and retrieve material completely anonymously. In Gnutella and MAC-SF, it is uncommon, yet possible for users to see what other users are searching for but this can stopped by the administrator by setting the option. Also, the retrieval of data is not anonymous at all in Gnutella. NSs are intelligent and able to identify "who is" downloading the data. But in MAC-SF it is not possible to see who is downloading the data. Obviously, Freenet and MAC-SF provide more privacy for users.

Freenet, Gnutella and MAC-SF create a decentralized network of NSs, but do so with different implementations. A NS handles and distributes data differently according to its own goal. Each has proved successful in its own way. In MAC-SF security is better than Gnutella and Freenet. Few private information in MAC-SF network is shared using X.509v3 certificate. Further MAC$\mathrm{SF}$ is designed for wireless heterogeneous networks, as per the nodes of the network application it groups and deputes a region a $\mathrm{RH}$.

\section{AdVANTAges OF MAC-SF}

The MAC-SF enables fast and cost-efficient deployment of self-managed distributed heterogeneous wireless system with high overall management cost, but with low management cost at each node. It implements a structured wireless concept which enables efficient resource management in distributed wireless system even during high rate of network whips. Further MAC-SF permits wireless computing/communication environment to every node by grouping the nodes in a region and deputing a node as RH. RH manages topology of the network and resource available in a MSC/BSC region. MAC-SF supports both device and code mobility. For task distribution it uses the MAP.

The management of high speed computing systems is highly complex and requires high-skilled administrators for their maintenance. MAC-SF permits large-scale large scale intelligent system deployment in distributed heterogeneous wireless networks without the need of cost-intensive supercomputing infrastructure. The approach is an evolutionary in the sense that it gives a new step towards the applications for heterogeneous wireless computing/communication environment into real-time services scenarios. In this system we have tried to improve the performance and incorporated new ideas for selection of desired wireless network before making handoff and other network servic 
International Journal on Computational Science \& Applications (IJCSA) Vol.5, No.5,October 2015

\section{CONCLUSION}

In this paper we have presented Movement Assisted Component Based Scalable Framework (MAC-SF) for distributed wireless systems. It is an evolutionary approach to wireless computing environment that fulfils most of the requirement heterogeneous wireless networks. MAC-SF enables fast and cost-efficient deployment of self-managed distributed heterogeneous wireless systems with high overall management cost. But it takes low management cost at node level. It implements a structured wireless concept which enables efficient resource management in heterogeneous wireless networks even during high rate of network beats. It permits distributed computing environment to every node by grouping the nodes in a region and deputing a node as $\mathrm{RH}$ and assumes whole network as group of regions. Each RH manages topology of the network and resources available in a MSC/BSC region. The network selection process is efficient. MACSF network selection process is tested through simulation and obtained result is satisfactory. We are in the process of testing the network selection in the real heterogeneous network. We are also studying the MAC-SF for Vertical Handover Decision making in cellular networks.

\section{REFERENCES}

[1] A Maccagnan., T. Vardanega, E. Feltriny, G. Valley, M. Rivaz, and N. Cannata, (2010) "MultiAgent System for the automated handling of experimental protocols in biological laboratories", In Proceedings of the 11th Workshop on Objects and Agents(WOA 2010), Dagli Oggetti Agli Agenti, A. Omicini and M. Viroli,( Eds.) Rimini, Italy, September 5-7,pp. 1-8.

[2] P. Yang, L. Tao, L Xu, and Z. Zhang, ( 2009) "Multiagent Framework for Bio-data Mining. In Rough Sets and Knowledge Technology,” P. Wen, Y. Li, L. Polkowski, Y. Yao, S. Tsumoto, and G. Wang,(Eds.) LNCS 5589: 200-207, Springer Berlin - Heidelberg

[3] G. S. Bhamra, A. K. Verma, and R. B. Patel,( 2015) "Agent Based Frameworks for Distributed Association Rule Mining: An Analysis”, International Journal in Foundations of Computer Science \& Technology, 5(1): 11-22

[4] G.S.Bhamra, A. K.Verma and R.B. Patel,(2015) "A Serial Computing Model of Agent Enabled Mining of Globally Strong Association Rules,” International Journal on Computational Sciences \& Applications, 5(3):77-104.

[5] J. J. Martínez, J. Garrigós, J. Toledo, and J. Manuel Ferrández, (2013) “An efficient and expandable hardware implementation of multilayer cellular neural networks," Neurocomputing, vol. 114, pp. 54 62.

[6] M. H. Bhuyan, D. K. Bhattacharyya, and J. K. Kalita,(2014) "Network anomaly detection: methods, systems and tools," IEEE Communications Surveys and Tutorials, vol. 16, no. 1, pp. 303-336.

[7] Kai Guo, and Zhijng Liu,( 2008) "A New Efficient Hierarchical Distributed P2P Clustering Algorithm", in IEEE Proceedings of the Fifth International Conference on Fuzzy Systems and Knowledge Discovery, pp. 352-355.

[8] Lijiang Chen, Bin Cui, Hua Lu, Linhao Xu, and Quanqing Xu,( 2008) "iSky: Efficient and Progressive Skyline Computing in a Structured P2P Network", in the IEEE Proceedings of the 28th International Conference on Distributed Computing Systems, pp. 160-169.

[9] Kai Guo, and Zhijng Liu,(2008) "A New Efficient Hierarchical Distributed P2P Clustering Algorithm", in IEEE Proceedings of the Fifth International Conference on Fuzzy Systems and Knowledge Discovery, pp. 352-355.

[10] Freenet project's Official Website Homepage. http://freenetproject.org/index.html

[11] Baihua Zheng et. al.,(2004) "On semantic caching and Query Scheduling for Mobile NearestNeighborSearch", in Proceedings of, Wireless Networks vol.10, pp. 653-664.

[12] Ming Xu, Shuigeng Zhou, Jihong Guan and Xiaohua Hu,(2010) "A path-traceable query routing mechanism for search in unstructured peer-to-peer networks". Journal of Network and Computer Application, 33(2): 115-127.

[13] Amos Korman, Amos, Shay Kutten, and Toshimitsu Masuzawa(2011). "Fast and compact self stabilizing verification, computation, and fault detection of an MST."Proceedings of the 30th annual 
International Journal on Computational Science \& Applications (IJCSA) Vol.5, No.5,October 2015

ACM SIGACT-SIGOPS symposium on Principles of distributed computing, San Jose, California,June 6-8, pp. 311-320.

[14] R. B. Patel and K. Garg,( 2004) “A New Paradigm for MA Computing”, WSEAS Transaction on Computers, 1(3): 57-64.

[15] J. al-Jaroodi, N. Mohamed, Hong Jiang and D. Swanson,(2003) "A Middleware Infrastructure for Parallel and Distributed Programming Models on Heterogeneous Systems", IEEE Transactions on Parallel and Distributed Systems, Special Issue on Middleware, 14(11):1100-1111.

[16] J. Al-Jaroodi, N. Mohamed, Hong. Jiang, and D. Swanson, (2003) "An Overview of Parallel and Distributed Java for Heterogeneous Systems: Approaches and Open Issues”, Journal of Parallel and Distributed Computing Practices.

[17] G.H. Forman, and J. Zahorjan, (1994) "The Challenges of Mobile Computing", IEEE Computer, 27(4): 38-47.

[18] D. Chess, B. Grosof, C. Harrison, D. Levine, C. Parris, and G. Tsudik,(1995) "Itinerant agents or mobile computing", IEEE Personal Communications Magazine, 2, pp. 34-49.

[19] T. Imielinsky, and B.R. Badrinath,(1994) "Wireless Computing: Challenges in Data Management", Communication of the ACM, 37(10): 18-28.

[20] Milojicic, D.,(1999), Trend Wars MA Applications, IEEE Concurrency, 7(3): 80 -90.

[21] Kendall, E.A., Krishna, P.V., Suresh C.B., and Pathak, C.V., (2000) “An Application Framework for Intelligent and MAs", ACM Computing Surveys, 32(1es).

[22] S. Lipperts, and A. Park, (1999) "An Agent Based Middleware: A Solution for Terminal and User Mobility”, Computer Networks, pp. 2053-2062.

[23] Nouha Oualha \& Jean Leneutre \& Yves Roudier,(2011) "Verifying remote data integrity in peer-topeer data storage: A comprehensive survey of protocols", Peer-to-Peer Networking Application, Vol. 4, pp. 1-11, (Springer)

[24] Kassar, Meriem, Brigitte Kervella, and Guy Pujolle, (2008) "An overview of vertical handover decision strategies in heterogeneous wireless networks." Computer Communications 31, no. 10: 2607-2620.

[25] Tuncer, Hasan, Sumita Mishra, and Nirmala Shenoy, (2012) "A survey of identity and handoff management approaches for the future Internet." Computer communications 36, no. 1: 63-79.

[26] Yan, Xiaohuan, Y. Ahmet Şekercioğlu, and Sathya Narayanan, (2010) "A survey of vertical handover decision algorithms in Fourth Generation heterogeneous wireless networks." Computer Networks 54, no. 11: 1848-1863.

[27] Márquez-Barja, Johann, Carlos T. Calafate, Juan-Carlos Cano, and Pietro Manzoni, (2011) "An overview of vertical handover techniques: Algorithms, protocols and tools." Computer Communications 34, no. 8: 985-997.

[28] Zekri, Mariem, Badii Jouaber, and Djamal Zeghlache, (2012) "A review on mobility management and vertical handover solutions over heterogeneous wireless networks." Computer Communications 35, no. 17: 2055-2068.

[29] Boukerche, Azzedine, Beguean Turgut, Nevin Aydin, Mohammad Z. Ahmad, Ladislau Bölöni, and Damla Turgut, (2011) "Routing protocols in ad hoc networks: A survey." Computer Networks 55, no. 13: 3032-3080.

[30] Shi, Fei, Keqiu Li, and Yanming Shen, (2010) "Seamless handoff scheme in Wi-Fi and WiMAX heterogeneous networks." Future Generation Computer Systems 26, no. 8: 1403-1408.

[31] Piamrat, Kandaraj, Adlen Ksentini, Jean-Marie Bonnin, and César Viho, (2011) "Radio resource management in emerging heterogeneous wireless networks."Computer Communications 34, no. 9: 1066-1076.

[32] Yan, Xiaohuan, Nallasamy Mani, and Y. Ahmet Şekercioğlu, (2008) "A traveling distance prediction based method to minimize unnecessary handovers from cellular networks to WLANs." Communications Letters, IEEE 12, no. 1: 14-16.

[33] Yang, Kemeng, Iqbal Gondal, Bin Qiu, and Laurence S. Dooley, (2007) "Combined SINR based vertical handoff algorithm for next generation heterogeneous wireless networks." In Global Telecommunications Conference, 2007. GLOBECOM'07. IEEE, pp. 4483-4487. IEEE.

[34] Ahmed, Atiq, Leïla Merghem-Boulahia, and Dominique Gaïti, (2010) "Cooperative agent based vertical handover scheme for heterogeneous networks." In Telecommunications (AICT), 2010 Sixth Advanced International Conference on, pp. 410-415. IEEE. 
International Journal on Computational Science \& Applications (IJCSA) Vol.5, No.5,October 2015

[35] Iraqi, Youssef, and R. Baoutaba. "Handoff and call dropping probabilities in wireless cellular networks, (2005) " In Wireless Networks, Communications and Mobile Computing, 2005 International Conference on, vol. 1, pp. 209-213. IEEE.

[36] Yang, Kemeng, Iqbal Gondal, Bin Qiu, and Laurence S. Dooley, (2007) "Combined SINR based vertical handoff algorithm for next generation heterogeneous wireless networks." In Global Telecommunications Conference, 2007. GLOBECOM'07. iEEE, pp. 4483-4487. IEEE.

[37] Hasswa, Ahmed, Nidal Nasser, and Hossam Hassanein,( 2006) "Tramcar: A context-aware crosslayer architecture for next generation heterogeneous wireless networks." In Communications, 2006. ICC'06. IEEE International Conference on, vol. 1, pp. 240-245. IEEE.

[38] E. P. Markatos,(2002) "Tracing a large-scale peer to peer system: An hour in the life of Gnutella", in CCGRID '02: Proceedings of the 2nd IEEE/ACM International Symposium on Cluster Computing and the Grid, pp. 65, Washington, DC, USA, IEEE Computer Society.

[39] M. Ripeanu and I. T. Foster,(2002) "Mapping the Gnutella network: Macroscopic properties of largescale peer-to-peer systems", in Proceedings of the IPTPS '01: Revised Papers from the 1st International Workshop on Peer-to-Peer Systems, pp. 85-93, London, UK. Springer-Verlag.

[40] Bar-Noy, I. Kessler, and M. Sidi,(1995) "Mobile Users: To update or not to update?" ACM-baltzer J. Wireless Networks Vol. 1, No. 2 175-186.

[41] Kimberly Keeton, Bruce A. Mah, Srinivasan Seshan, Randy H. Katz, and Domenico Ferrari,(1993) "Providing connection oriented network services to mobile NSs," in the Proceedings of the USENIX Mobile \& Location-Independent Computing Symposium, Cambridge, Massachusetts, August 2-3, pp. 1-32.

[42]Tavana, M., \& Hatami-Marbini, A. (2011). A group AHP-TOPSIS framework for human spaceflight mission planning at NASA. Expert Systems with Applications, 38(11), 13588-13603.

\section{Authors}

Sanjeev Prakash is a Ph.D. scholar in the Department of Computer Science and Engineering, Sant Longowal Institute of Engineering and Technology, Longowal, India. He did M. Tech. in Computer Science and Engineering from Kurukshetra University, Kurukhetra, India in 2008. His research interest in the area of ad hoc and wireless networks.

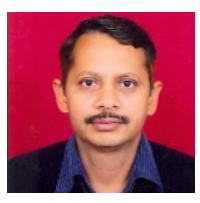

Dr. Ram Bahadur Patel is currently working as Professor at Department of Computer Science \& Engineering, Chandigarh College of Engineering and Technology, Chandigarh. He received $\mathrm{PhD}$ from IIT Roorkee in Computer Science and Engineering, PDF from Highest Institute of Education, Science \& Technology (HIEST), Athens, Greece, MS (Software Systems) from BITS Pilani and B. E. in Computer Engineering from M. M. M. Engineering College, Gorakhpur, UP. Dr. Patel is in teaching and research since 1991. He has supervised $40 \mathrm{M}$. Tech, $7 \mathrm{M}$. Phil. and 12 $\mathrm{PhD}$ Thesis. He is currently supervising $4 \mathrm{PhD}$ students. He has published 140 research papers in International/National Journals and Refereed International Conferences. He has written 7 text books for engineering courses. He is member of ISTE (New Delhi), IEEE (USA). He is a member of various International Technical Committees and participating frequently in International Technical Committees in India and abroad. His current research interests are in Mobile \& Distributed Computing, MA Security and Fault Tolerance and Sensor Network.

Dr. V.K. Jain is Director, Sant Longowal Institute of Engineering and Technology, Longowal, India. He did his B. Tech., M. Tech. and Ph.D. from REC, Kurukeshetra in the year 1977, 1979 and 1994, respectively. He has guided 40 M. Tech., 3 Ph.D. thesis and presently he is supervising $8 \mathrm{Ph} . \mathrm{D}$. scholars. Dr. Jain published more than 100 papers in International/National journals and conferen ces. His area of research are reliability, biomedical engineering ad hoc networks, Image Processing and Natural Language processing.

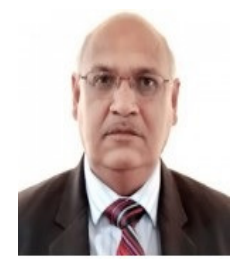

